\title{
Methods of Measuring the Resistivities of Anisotropic Conducting Media in Situ
}

\author{
Stanley Rush ${ }^{1}$
}

\author{
(April 16, 1962)
}

\begin{abstract}
Several static boundary value problems involving anisotropic media have been solved and the results are presented here. The solutions have been applied to simple electrode configurations to obtain formulas for in situ determinations of the resistivities of anisotropic conducting media. The boundaries treated include infinite, semi-infinite, slab and spherical shapes. The media are anisotropic with a common resistivity in two orthogonal directions and a lower resistivity in the third orthogonal direction.
\end{abstract}

\section{Introduction}

In the following, field solutions and measurement equations suitable for finding the electrical resistivities of anisotropic conducting media and analogous parameters are derived for several boundary configurations. The results have been applied to the measurement of the resistivities of living muscle tissue in situ but they may also be used for the measurement of the electrical properties of other anisotropic media, for example; the conductivities of earth formations and semiconductors and the dielectric constants of uniaxial crystals. The measured values of muscle resistivity and descriptions of the physical apparatus will be reported elsewhere.

The anisotropy of muscle is correlated with the directions of muscle fibers. These appear to consist, electrically speaking, of high resistivity tubular membranes surrounded within and without with low resistivity fluids. The membranes have a common orientation in a given muscle, yielding one direction of low resistivity parallel to the fibers and two directions of high resistivity transverse to the fibers, To maintain the muscle in its natural state during the measurements, the measuring electrodes were placed on the muscle surface rather than in the tissue interior. With one exception, the equations developed here reflect these conditions.

When applied to the measurements of skeletal muscle in living animals the precision of the methods to be described were limited by the inhomogeneities of the tissue and the difficulties of obtaining exact alinement of the electrode structure with the axes of anisotropy.

\section{Potential Field of a Spherical Current Source in an Infinite Anisotropic Medium}

The potential field of a spherical current source, and its specialization to a point source, in a linear, homogeneous, infinite, anisotropic, conducting medium with sink at infinity, forms the basis for all the results to be derived. For anisotropic media having one direction of high conductivity and two directions of low conductivity, the solution to the stated problem can be obtained by a procedure given by Smythe. ${ }^{2}$

The electrostatic equation of continuity can be written in rectangular coordinates as

$$
-\nabla \cdot \mathbf{J}=\sigma_{x} \frac{\partial^{2} V}{\partial x^{2}}+\sigma_{y} \frac{\partial^{2} V}{\partial y^{2}}+\sigma_{z} \frac{\partial^{2} V}{\partial z^{2}}=0
$$

in which $\mathbf{J}$ is the current density, $V$ the potential and $\sigma_{x}, \sigma_{y}$, and $\sigma_{z}$ are the conductivities in the $x, y$, and $z$ directions respectively. The origin of coordinates is taken at the center of the sphere, figure 1 . In the medium of interest, $\sigma_{x}=\sigma_{z}=\sigma_{l}$ and $\sigma_{y}=\sigma_{h} . \alpha^{2}$ is defined as $\sigma_{l} / \sigma_{h} \leq 1$. Equation (1) becomes Laplace's equation in a new coordinate system in which $\mu=\alpha y$ as

${ }^{2}$ Smythe, W. R., Static and Dynamic Electricity, 2d ed. (McGraw-Hill Book Co., 1950), p. 248 .

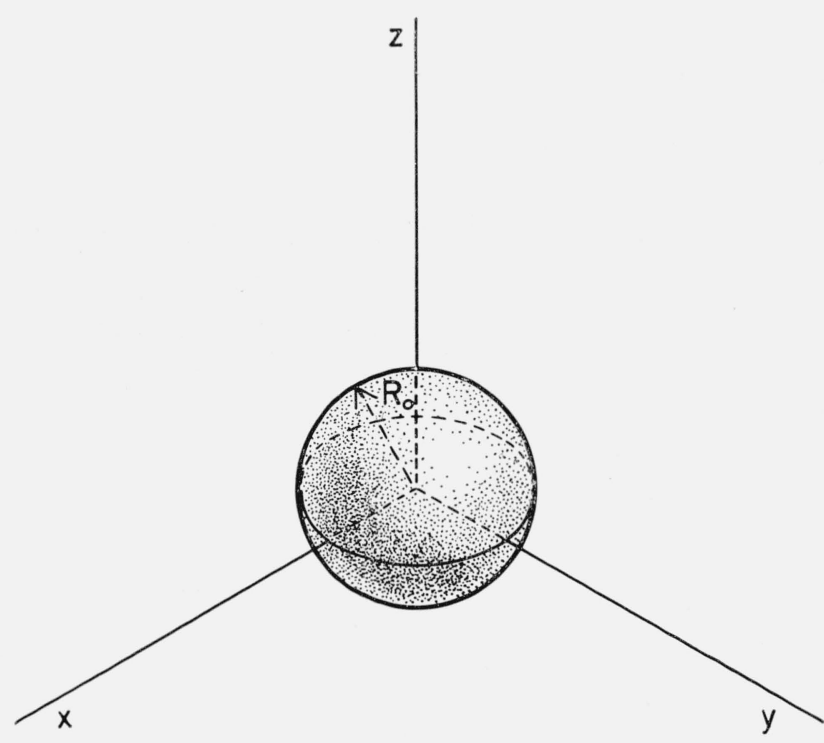

FIGURE 1. Coordinate relations for calculating the potential of a spherical electrode in an anisotropic medium. 


$$
\frac{\partial^{2} V}{\partial x^{2}}+\frac{\partial^{2} V}{\partial \mu^{2}}+\frac{\partial^{2} V}{\partial z^{2}}=0
$$

The spherical electrode surface defined by $x^{2}+y^{2}$ $+z^{2}=R_{0}^{2}$ becomes, in the $x, \mu, z$ coordinates, an oblate spheriod defined by

$$
\frac{x^{2}}{R_{0}^{2}}+\frac{\mu^{2}}{\alpha^{2} R_{0}^{2}}+\frac{z^{2}}{R_{0}^{2}}=1
$$

Smythe shows that the solution to eq (2) may be expressed as

$$
\begin{aligned}
V=V_{0} \int_{\nu}^{\infty} \frac{d \nu}{\left(a^{2}+\nu\right)\left(b^{2}+\nu\right)^{1 / 2}} \\
\quad\left[\int_{0}^{\infty} \frac{d \nu}{\left(a^{2}+\nu\right)\left(b^{2}+\nu\right)^{1 / 2}}\right]^{-1}
\end{aligned}
$$

in which $\nu$ is the confocal ellipsoid parameter of the equation

$$
\frac{x^{2}}{\left(a^{2}+\nu\right)}+\frac{\mu^{2}}{\left(b^{2}+\nu\right)}+\frac{z^{2}}{\left(a^{2}+\nu\right)}=1 .
$$

At the electrode surface defined by $\nu=0$, eq (5) reduces to eq (3). Hence $a=R_{0}$ and $b=\alpha R_{0}$. By changing the variables of integration in eq (4) to $\nu^{\prime}=a^{2}+\nu$, the integrals can be evaluated by Dwight ${ }^{3}$ 192-11, giving.

$$
V=V_{0} \frac{\left[\tan ^{-1} \frac{\left(b^{2}-a^{2}+\nu^{\prime}\right)^{1 / 2}}{\left(a^{2}-b^{2}\right)^{1 / 2}}\right]_{\nu^{\prime}}^{\infty}}{\left[\tan ^{-1} \frac{\left(b^{2}-a^{2}+\nu^{\prime}\right)^{1 / 2}}{\left(a^{2}-b^{2}\right)^{1 / 2}}\right]_{a^{2}}^{\infty}}
$$

which reduces, upon insertion of the limits, to

$$
V=\frac{V_{0}}{\sin ^{-1}\left(1-\alpha^{2}\right)^{1 / 2}} \tan ^{-1} \frac{\left(a^{2}-b^{2}\right)^{1 / 2}}{\left(b^{2}+\nu\right)^{1 / 2}} .
$$

Solving eq (5) for $\nu$ and substituting in eq (7), yields the potential outside the spherical electrode in the original coordinates;

$$
\begin{aligned}
V= & \frac{V_{0}}{\sin ^{-1}\left(1-\alpha^{2}\right)^{1 / 2}} \times \sin ^{-1} \\
& \frac{(2 A)^{1 / 2}}{\left[\rho^{\prime 2}+\alpha^{2} y^{2}+A+\left[\left(\alpha^{2} y^{2}+\rho^{\prime 2}-A\right)^{2}+4 \alpha^{2} y^{2} A\right]^{1 / 2}\right]^{1 / 2}}
\end{aligned}
$$

in which $A=R_{0}{ }^{2}\left(1-\alpha^{2}\right)$ and $\rho^{\prime}=\left(x^{2}+z^{2}\right)^{1 / 2}$. Equation (8) also applies to a hemispherical electrode in a semi-infinite conducting medium; i.e., $z>0$ an insulator in figure (1).

The point source solution is of particular interest and is found from eq (8) by assuming $y$ and $\rho^{\prime}$ very large as

$$
V=\frac{V_{0}}{\sin ^{-1}\left(1-\alpha^{2}\right)^{1 / 2}} \frac{(A)^{1 / 2}}{\left(\rho^{\prime 2}+\alpha^{2} y^{2}\right)^{1 / 2}} .
$$

Equation (9) can also be written in terms of the total current, $I$, leaving the electrode by integrating the normal component of $\mathbf{J}$ over a closed surface. By

${ }^{3}$ Dwight, H. B., Tables of Integrals and Other Mathematical Data, (MacMillan Co., New Ýork), 3d edition. expressing $\mathbf{J}$ in terms of $V$ and integrating over a cube of edge $2 w$, symmetrically disposed about the origin, we have

$$
\begin{aligned}
I= & \frac{V_{0}(A)^{1 / 2}}{\sin ^{-1}\left(1-\alpha^{2}\right)^{1 / 2}}\left[\sigma_{h} w \int_{-w}^{w} \int_{-w}^{w} \frac{d x d y}{\left(x^{2}+w^{2}+\alpha^{2} y^{2}\right)^{3 / 2}}\right. \\
& +\sigma_{h} w \int_{-w}^{w} \int_{-w}^{w} \frac{d y d z}{\left(w^{2}+z^{2}+\alpha^{2} y^{2}\right)^{3 / 2}} \\
& \left.+\alpha^{2} \sigma_{l} w \int_{-w}^{w} \int_{-w}^{w} \frac{d x d z}{\left(x^{2}+y^{2}+\alpha^{2} w^{2}\right)^{3 / 2}}\right] .
\end{aligned}
$$

The bracket reduces to $4 \pi\left(\sigma_{l} \sigma_{h}\right)^{1 / 2}$. For a semiinfinite medium, $\sigma_{l}$ and $\sigma_{h}=0$ where $z>0$ and the bracket becomes $2 \pi\left(\sigma_{l} \sigma_{l}\right)^{1 / 2}$. Thus, in the latter case, eq (9) becomes

$$
V=I\left[4 \pi^{2} \sigma_{l} \sigma_{h}\left(\rho^{\prime 2}+\alpha^{2} y^{2}\right)\right]^{-1 / 2} .
$$

The measurement of $\sigma_{x}, \sigma_{y}$, and $\sigma_{z}$ of a semiinfinite anisotropic medium can conveniently be made by applying eq (10) to a simple electrode arrangement. Assume the semi-infinite insulating boundary (usually air), figure 2 , is at the $z=0$ plane; i.e., the conductivities are zero for $\mathrm{z}>0$. Medium (1) is assumed anisotropic such that $\sigma_{x}=\sigma_{z}=\sigma_{l}$ and $\sigma_{y}=\sigma_{h}$. The electrodes are shown schematically in figure 2 and consist of four, equally spaced, approximately spherical, conductors of small size. The dimensions of the electrodes must be such as to make eq (10) a good approximation to eq (8) at a distance, $a$, from the origin; and to eliminate for practical purposes, perturbations by the remaining conductors of the potential field of the source or sink. The potential difference between points $g$ and $h$ due to electrode $e$ can be found from eq (10). Noting that electrode $f$ doubles this potential differ-

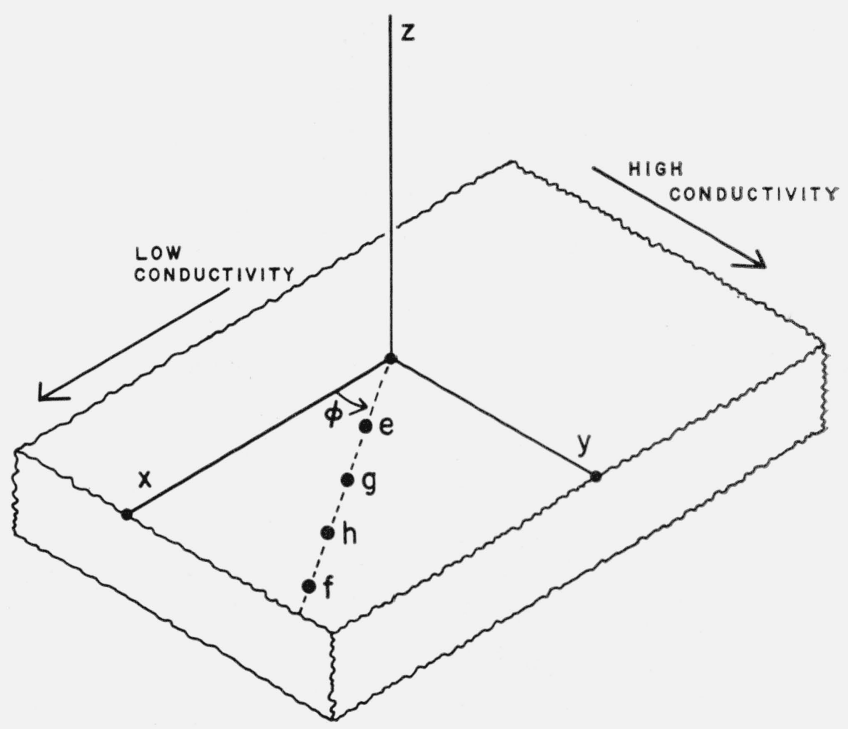

FIGURE 2. Arrangement of four point-electrodes for measuring the resistivities of an anisotropic, semi-infinite, conducting medium. 
ence and expressing the result in polar coordinates, see figure 2 , the potential difference, $V_{g h}$, can be written

$$
V_{g h}(\phi)=I\left[4 \pi^{2} \sigma_{l} \sigma_{h}\left(\cos ^{2} \phi+\alpha^{2} \sin ^{2} \phi\right)\right]^{-1 / 2} .
$$

Two measurements of $V_{g h}$, at $\phi=0$ and $\phi=\pi / 2$, give $\sigma_{l}$ and $\sigma_{h}$ from eq (11) as follows:

$$
\begin{aligned}
& \sigma_{l}=I\left[2 \pi a V_{g h}(\pi / 2)\right]^{-1} \\
& \sigma_{h}=\sigma_{l}\left[V_{g h}(\pi / 2) / V_{g h}(0)\right]^{2} .
\end{aligned}
$$

\section{Measurement of the Resistivities of an Anisotropic Conducting Slab}

It may happen that the anisotropic material of interest, $z<0$, figure 2 , has a limited extension in the negative $z$ direction; the shape being referred to as a 'slab.' When slab thickness, $h$, is less than twice the electrode spacing, $a$, the effects of the boundary may make eqs (12) and (13) poor approximations to the slab resistivities.

If the boundary at $z=-h$ is either a perfect conductor or an insulator, however, exact mathematical expressions for the resistivity of the slab as a function of quantities measurable with the electrodes of figure 2 can be derived. To obtain these expressions, we find first the following potential fields:

The potential on the surface of an anisotropic conducting slab arising from a point current source on the surface and sink at infinity. The slab has a high conductivity in the $y$ direction and low conductivity in the $x$ and $z$ directions, is bounded at $z=0$ by an insulator and at $z=-h$ by (a) an insulator and (b) a perfect conductor.

Generalizations of the method of solution to include arbitrary field point and source locations in the slab will be apparent.

Figure 3a represents a section of the given problem in the $y z$ plane; figure $3 \mathrm{~b}$, a system of images located in an infinite homogeneous medium having the slab conductivities. The image system satisfies the boundary conditions at $z=0$ and $z=-h$ and can therefore be used to obtain the field within the slab. As shown in figure $3 \mathrm{~b}$ the images extend to infinity in the plus and minus $z$ directions, spaced a distance $2 h$ apart. They are equal in strength to the source plus its image in the $z=0$ plane; i.e., $2 I$ and are of the same sign if $z=-h$ is an insulator and alternate in sign if $z=-h$ is a perfect conductor. The potential on the slab surface can be written by inspection using the principle of superposition and eq (10) suitably modified for a source not at the origin. The resulting potential on the surface $z=0$, is

$$
\begin{aligned}
V=\frac{I}{2 \pi\left(\sigma_{l} \sigma_{h}\right)^{1 / 2}} & {\left[\left(x^{2}+\alpha^{2} y^{2}\right)^{-1 / \mathbf{2}}\right.} \\
& \left.+2 \sum_{n=1}^{\infty}( \pm 1)^{n}\left[x^{2}+\alpha^{2} y^{2}+(2 n h)^{2}\right]^{-1 / 2}\right]
\end{aligned}
$$

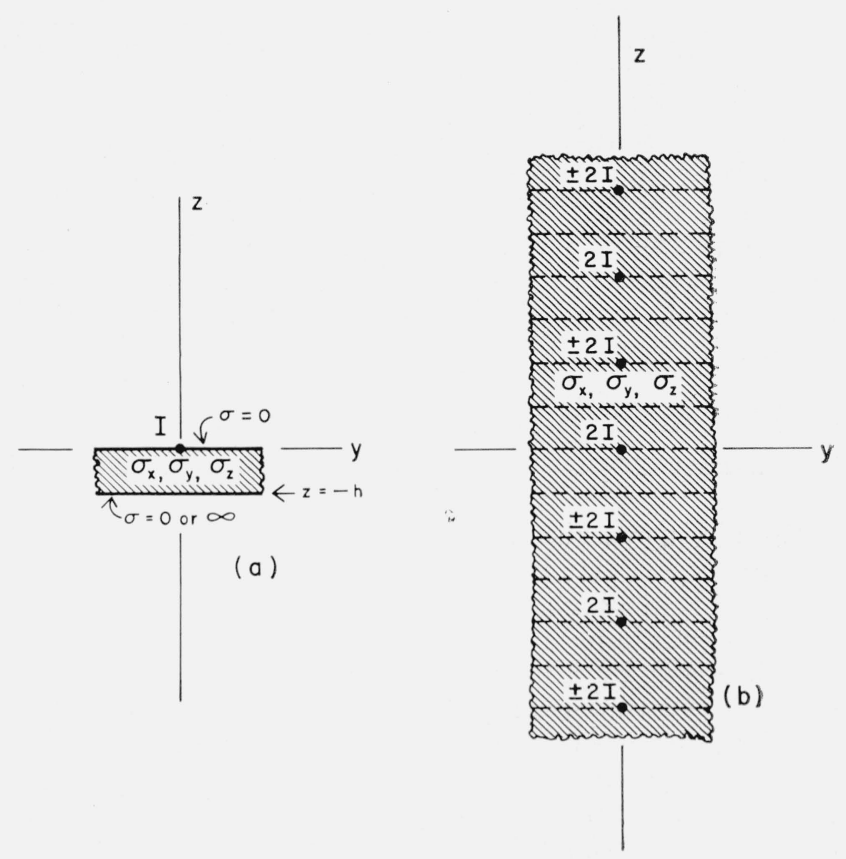

FIGURE 3. (a) yz-plane section of an anisotropic slab of thickness, $\mathrm{h}$, with current source at the origin.

(b) System of images of strength, $\pm 2 \mathrm{I}$, which match boundary conditions at slab surface.

Images are infinite in number and spaced a distance, $2 h$, apart. The minus signs appear at alternate images when $z<-h$ is a perfect conductor.

The plus sign applies when the $z=-h$ boundary is an insulator and the minus sign when it is a perfect conductor, each term in the summation being an image contribution. ${ }^{4}$

Again considering the electrodes of figure 2, the potential differences measured on the slab surface at $\phi=0 \quad(y=0)$ and $\phi=\pi / 2 \quad(x=0)$ can be expressed as

$$
\begin{array}{r}
V_{g h: y=0}=\frac{I}{2 \pi a\left(\sigma_{l} \sigma_{h}\right)^{1 / 2}}\left[1+2 \sum_{1}^{\infty}( \pm 1)^{n}\left(2\left[1+(2 n h / a)^{2}\right]^{-1 / 2}\right.\right. \\
\left.\left.-\left[1+(n h / a)^{2}\right]^{-1 / 2}\right)\right] ; \quad
\end{array}
$$

$$
\begin{aligned}
V_{g h: x=0}=\frac{I}{2 \pi \sigma_{l} a}\left[1+2 \sum_{1}^{\infty}(\right. & \pm 1)^{n}\left(2\left[1+(2 n h / \alpha a)^{2}\right]^{-1 / 2}\right. \\
& \left.\left.-\left[1+(n h / \alpha a)^{2}\right]^{-1 / 2}\right)\right] .
\end{aligned}
$$

The conductivities $\sigma_{l}$ and $\sigma_{h}$ can conveniently be found from the measurements of $V_{g h}$. Thus, taking the ratio of the equations in (15) we have

$$
\frac{V_{g h: y=0}}{V_{g h: x=0}}=\frac{\alpha B}{B(\alpha)}
$$

in which $B$ and $B(\alpha)$ are the terms in square brackets in eqs (15a) and (15b) respectively. Plots of $B(\alpha)$ as a function of $h / \alpha a$ (note that $B=B(\alpha)$ if $\alpha=1$ )

\footnotetext{
${ }^{4}$ Although eq (14) diverges when the plus sign is used, eqs (15) do not.
} 
can be found in the geophysical literature. ${ }^{5}$ The graphs are reproduced here in figure 4 with minor modifications. Using eq (16) and figure 4 it is possible to obtain $\alpha$ quickly by successive approximations. As is apparent from figure 4, when $a / h>1.5$, the asymptotic formula, $B=1.39(a / h)$, can be used; and when $\alpha a<.25, B(\alpha)$ can be approximated as unity. Once $\alpha=\left(\sigma_{l} / \sigma_{h}\right)^{1 / 2}$ is found, eqs (15), together with figure 4 can be used to find $\sigma_{l}$ and $\sigma_{h}$.

\section{Measurement of the Resistivities of an Anisotropic Conducting Hemisphere}

Should the anisotropic material have a spherical boundary, it is possible to obtain measurement equations in some instances. In particular, closed form expressions can be derived for the potential distribution in the hemisphere of figure 5. The source is a point current electrode arbitrarily located on the sphere surface and the sink a perfectly conducting meridian plane. The hemisphere has a high conductivity, $\sigma_{h}$, in the $\phi$ direction and low conductivity, $\sigma_{l}$, in the $R$ and $\theta$ directions. Approximate expressions, based on a four-electrode measurement, can be derived for $\sigma_{h}$ and $\sigma_{l}$ from the potential field.

This problem arose in measuring the resistivities of heart muscle. The muscle fibers were assumed to be wrapped around an axis in a manner roughly analogous to string wound into a ball. It should be noted that the field expression is unchanged if the hemisphere is replaced by a sphere and the conducting plane electrode by a point sink at the location of an image of the source in the plane.

As shown in figure 5 , the point electrode is on the surface at $R=C, \theta=\theta_{1}, \phi=\phi_{1}$ and the plane electrode is at $\phi= \pm \pi / 2$. The equation of continuity can, with an appropriate coordinate transformation, be expressed as Laplace's equation in spherical coordinates. The result is given in eq (17) in which $\phi^{\prime}=\alpha \phi$.

$$
\begin{array}{r}
\nabla^{2} V=\frac{1}{R^{2}} \frac{\partial}{\partial R}\left(R^{2} \frac{\partial V}{\partial R}\right)+\frac{1}{R^{2} \sin \theta} \frac{\partial}{\partial \theta}\left(\sin \theta \frac{\partial V}{\partial \theta}\right) \\
+\frac{1}{R^{2} \sin ^{2} \theta} \frac{\partial^{2} V}{\partial \phi^{\prime 2}}=0
\end{array}
$$

The boundary value problem in the new $\left(R, \theta, \phi^{\prime}\right)$ coordinate system is that of a point current source at $R=C, \theta=\theta_{1}, \phi^{\prime}=\alpha \phi_{1}$ on a wedge-shaped section of a sphere, and a sink at constant-potential intersecting (internal angle $\alpha \pi$ ) planes, figure 6 .

An image solution for the potential field in the wedge exists if $1 / \alpha$ is an integer; i.e., if the internal wedge angle is a submultiple of $\pi$. Sources and sinks placed on the surface of a sphere as shown in figure 7 will make the planar wedge surfaces equipotentials and satisfy the remaining boundary conditions as well. More general results arising from

5 Hummel, J. N., AIME Tech. Publ. No. 418 and Heiland, C. A., Geophysical Exploration, (Prentice-Hall, New York, 1940), p. 716

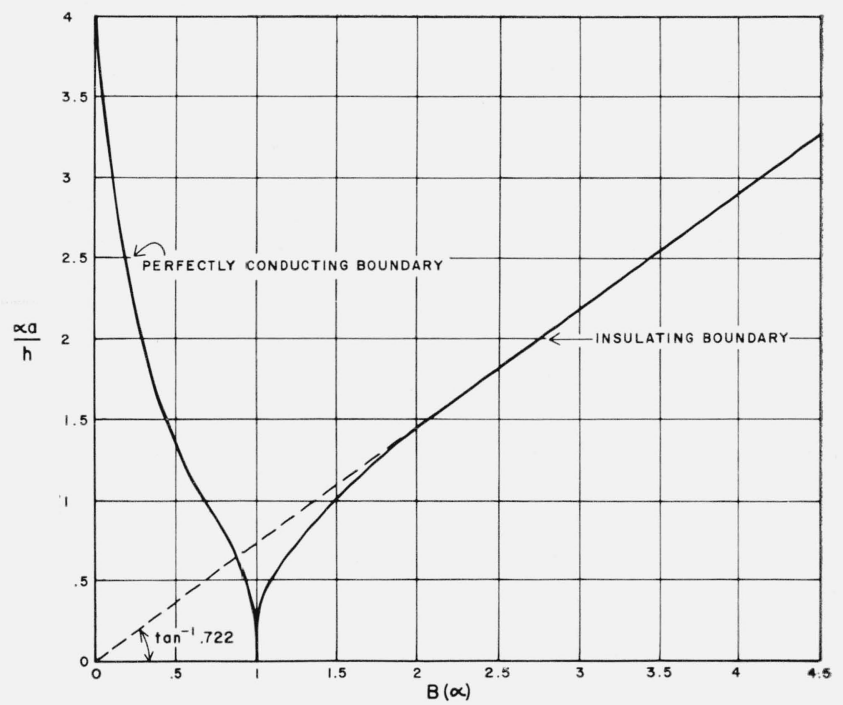

Figure 4. Graphs used to solve for $\alpha$ in eq 16 (after Hummel).

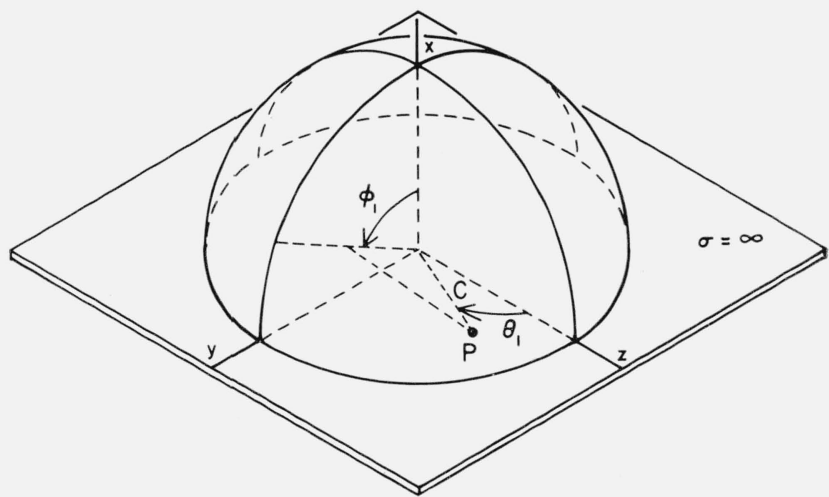

FIGURE 5. Anisotropic conducting hemisphere on a perfectly conducting meridian plane.

Electrodes are on plane and at point, $P$, on the sphere surface. The hemisphere has a high conductivity, $\sigma_{h}$, in the $\phi$ direction and equal but lower conductivities, $\sigma_{l}$, in the $\theta$ and $R$ directions, $C$ is the radius of the hemisphere.

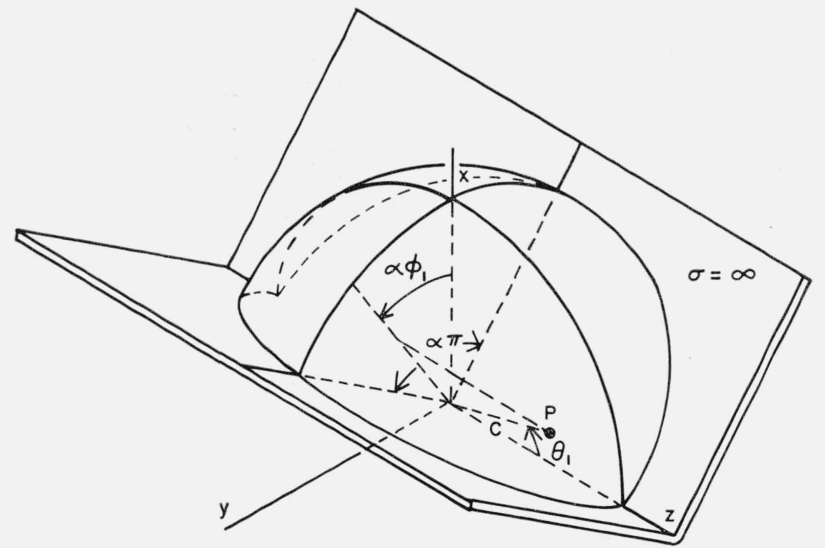

FIGURE 6. Hemisphere of figure 5 transformed to new coordinates in which $\phi^{\prime}=\alpha \dot{\phi}$. 
a source inside the wedge can be obtained by similar reasoning.

The potential due to a source-sink pair arbitrarily located in a sphere has been given by Frank. ${ }^{6}$ His result, specialized to the case in which the source and sink as well as the field point are on the sphere surface and extended by superposition to $q$ sourcesink pairs, is the desired equation for the potential arising from the system of images shown in figure 7 . This extended result, which gives the potential on the wedge, is presented as eq (18).

$$
V=k \sum_{i=1}^{2 q} \pm\left(\frac{1}{r_{i}^{\prime}}-\frac{1}{2 C} \ln \left[r_{i}^{\prime}+C\left(1-\cos \psi_{i}^{\prime}\right)\right]\right)
$$

$C=$ the radius of the sphere.

$r_{i}^{\prime}=$ the chord distance from the $i$ th source (or sink) point to the field point in the $\left(R, \theta, \phi^{\prime}\right)$ coordinates.

$=C\left[2\left(1-\cos \Psi_{i}^{\prime}\right]^{1 / 2}\right.$.

$\Psi_{i}^{\prime}=$ the angle formed at the origin by the radius vector to the field point $\left(C, \theta, \phi^{\prime}\right)$ and the $i$ th source (or sink) point $\left(C, \theta_{i}, \phi_{i}^{\prime}\right)$.

$=\cos ^{-1}\left[\cos \theta \cos \theta_{i}+\sin \theta \sin \theta_{i} \cos \left(\phi^{\prime}-\phi_{i}^{\prime}\right)\right]$.

$k=1 / 2 \pi\left(\sigma_{l} \sigma_{h}\right)^{1 / 2}$, as can be shown by comparing eq (10) with eq (18) as $C \longrightarrow \infty$.

The potential field in the original coordinates is found by substituting $\alpha \phi$ for $\phi^{\prime}$ and $\alpha \phi_{i}$ for $\phi_{i}^{\prime}$ in eq (18).

When the conditions described below are satisfied, a convenient formula for determining approximate values of the conductivities $\sigma_{l}$ and $\sigma_{h}$, applies to the electrode configuration of figure 8 . In this case, electrode $e$ and electrode $f$ each give rise to a set of images. The electrodes are spaced along the arc of a circle of radius, $C$, and the potential-measuring electrodes are at distances $a$ and $2 a$ from the current

${ }^{6}$ Frank, E., J. of A ppl. Phys. 23, 1225, 1952.

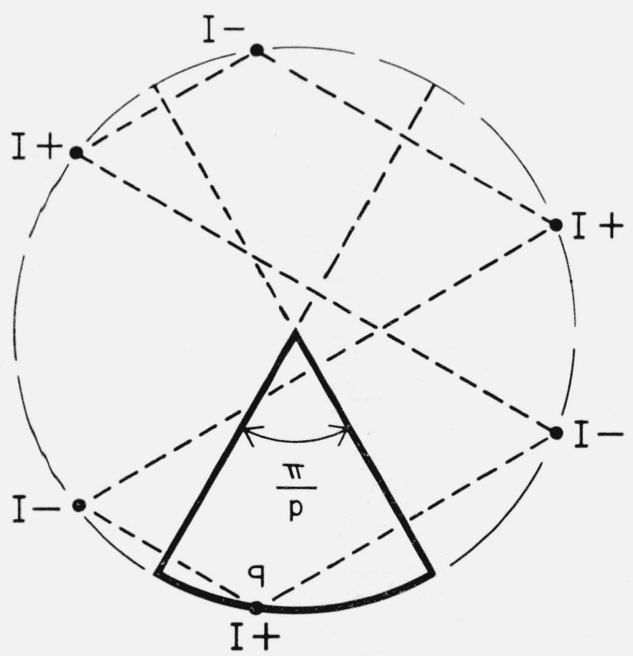

Figure 7. Latitudinal plane section of figure 6 showing image construction for $\mathrm{q}=3$.

The current, $I$, is shown entering at the point, $P$. The radius of the circular section is, $C \sin \theta_{1}$ electrodes. If the source is located far from the constant-potential sink plane, figure 5 ; i.e., in a region defined by $-\pi / 4<\phi<\pi / 4,3 \pi / 4<\theta<\pi / 4$, and the electrode spacing $a$ is small compared to the sphere radius, the contribution of the images to the potential difference measured by the electrodes of figure 8 can be reduced to the order of one percent. From (18) the potential differences, neglecting images, measured by electrodes $g$ and $h$, figure 8 , due to the current electrodes $e$ and $f$, reduce to convenient expressions at two particular electrode alinements; i.e., when the electrodes lie along a line of constant $\phi$ or constant $\theta$. In fact

$$
\begin{aligned}
& V_{g h: \text { cons } \tan t \phi}=\frac{I}{C \pi\left(\sigma_{h} \sigma_{l}\right)^{1 / 2}} \\
& \times\left[\left(2\left[1-\cos \left(\theta_{e}-\theta_{g}\right)\right]\right)^{-1 / 2}-\left(2\left[1-\cos \left(\theta_{e}-\theta_{h}\right)\right]\right)^{-1 / 2}\right. \\
& \left.+\frac{1}{2} \ln \frac{\left[2-2 \cos \left(\theta_{e}-\theta_{h}\right)\right]^{1 / 2}+\left[1-\cos \left(\theta_{e}-\theta_{h}\right)\right]}{\left[2-2 \cos \left(\theta_{e}-\theta_{g}\right)\right]^{1 / 2}+\left[1-\cos \left(\theta_{e}-\theta_{g}\right)\right]}\right]
\end{aligned}
$$

Here we have used $\theta_{e}$ for the polar angle of the source point, electrode $e$; and $\theta_{g}$ and $\theta_{h}$ for the polar angles of the field points, electrodes $g$ and $h$ respectively. It is clear from the form of eq (19) and the angular relations shown in figure 8 that the factor of two used in eq (19) correctly accounts for the effects of the sink electrode, $f$. Noting that the denominators of the first two terms in brackets on the right are chord lengths, $a$ and $2 a$ respectively, eq (19) becomes

$$
V_{g h: \text { constant } \phi}=\frac{I}{\pi\left(\sigma_{h} \sigma_{l}\right)^{1 / z}}\left(\frac{1}{2 a}+\frac{1}{2 C} \ln \frac{2(1+a / C)}{(1+a / 2 C)}\right) .
$$

In a similar way, when the electrodes are alined along a latitude line, and if the sines of angles $\alpha_{1}$ and $\alpha_{2}$, figure 8 , subtended at the sphere center by the current and potential electrodes are approximated by the angles, we have

$$
V_{g h: \operatorname{constant} \theta}=\frac{I}{\pi\left(\sigma_{h} \sigma_{l}\right)^{1 / 2}}\left(\frac{1}{2 \alpha a}+\frac{1}{2 C} \ln \frac{2(1+\alpha a / C)}{(1+\alpha a / 2 C)}\right) .
$$

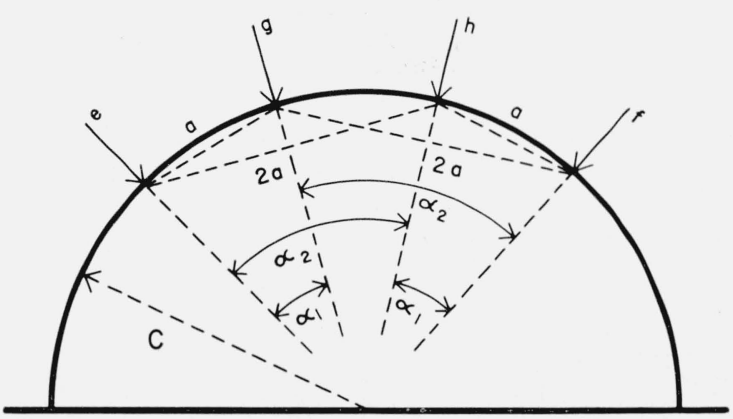

FIGURE 8. Electrode arrangement for measuring the resistivities of the hemisphere of figure 5 using eqs (20) and (21). 
In the ratio of eq (20) and eq (21), $\alpha$ is the only unknown and may be found by successive approximations; after which $\sigma_{l}$ and $\sigma_{h}$ can be obtained from either eq (20) or eq (21).

\section{Resistance Between Two Small Spheres in an Anisotropic Medium}

An "apparent resistivity" of an anisotropic medium has been obtained by comparing the resistance measured between two small spherical electrodes in an anisotropic medium with the resistance between similar electrodes in an isotropic medium of known resistivity. ${ }^{7}$ When the two resistances are equal, the apparent resistivity is defined to be the known isotropic resistivity. The apparent resistivity, in addition to being a function of $\sigma_{l}$ and $\sigma_{h}$, is a function of the orientation of the axis connecting the spheres. An approximate formula for this functional dependence will be derived. The coordinate relations are shown in figure 9 . A first approximation to the resistance, $\Omega$, between two spheres is well known to be ${ }^{8}$

$$
\Omega=2\left[V\left(R_{0}\right)-V(\mathbf{d})\right] / I
$$

in which $V\left(R_{0}\right)$ is the potential on the surface of the sphere of radius, $R_{0}$, centered at the origin and $V(\mathbf{d})$ is the potential due to the sphere at the origin at the center of the second sphere (at radius vector d), neglecting the perturbing effect of the second sphere. Rewriting eq (8) in polar coordinates with $x^{2}+y^{2}+z^{2}$ $=d^{2}$ and the constant $V_{0} / \sin ^{-1}\left(1-\alpha^{2}\right)^{1 / 2}$ as $I / 4 \pi$ $\left(\sigma_{l} \sigma_{h} A\right)^{1 / 2}$, we have

$$
V(\mathbf{d})=\frac{I}{4 \pi\left(\sigma_{h} \sigma_{l} A\right)^{1 / 2}} \sin ^{-1} \frac{R_{0}\left[2\left(1-\alpha^{2}\right)\right]^{1 / 2}}{K d},
$$

7 Schwan, H. P., and Kay, C. F., Circulation Research 4, 664 (1956). "Smythe, W. R., op. cit., p. 238.

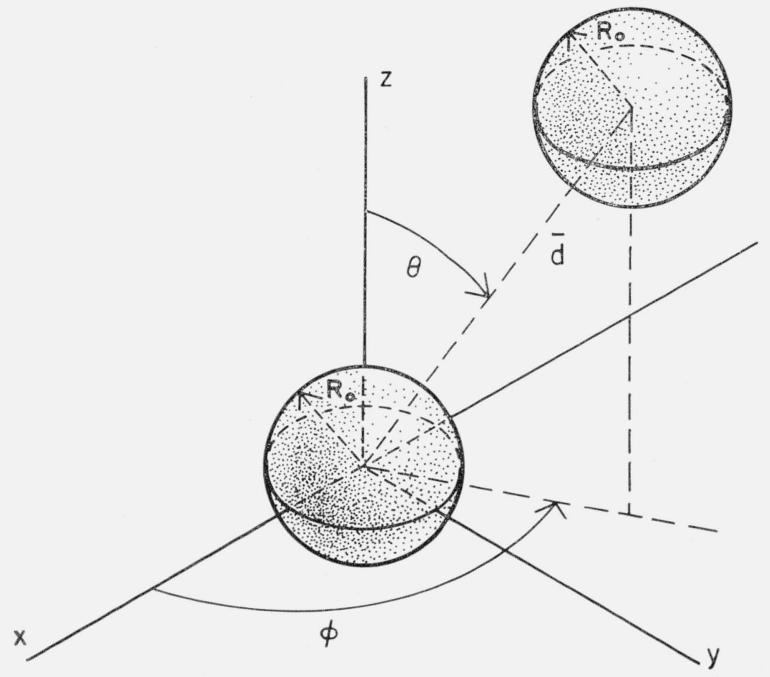

FIGURE 9. Coordinate relations for evaluating approximate resistance between two spheres of radii, $\mathrm{R}_{0}$, and center-tocenter distance, $\mathrm{d}$. in which

$$
\begin{aligned}
K= & {\left[1-\left(1-\alpha^{2}\right) \sin ^{2} \phi \sin ^{2} \theta+\left(\frac{R_{0}}{d}\right)^{2}\left(1-\alpha^{2}\right)\right.} \\
& +\left(\left[1-\left(1-\alpha^{2}\right) \sin ^{2} \phi \sin ^{2} \theta-\left(\frac{R_{0}}{d}\right)^{2}\left(1-\alpha^{2}\right)\right]^{2}\right. \\
& \left.\left.+4 \alpha^{2} \sin ^{2} \phi \sin ^{2} \theta\left(\frac{R_{0}}{d}\right)^{2}\left(1-\alpha^{2}\right)\right)^{1 / 2}\right]^{1 / 2} .
\end{aligned}
$$

$V\left(R_{0}\right)$ is a special case of eq (23) obtained by letting $d=R_{0}$ with the result

$$
V\left(R_{0}\right)=\frac{I \sin ^{-1}\left(1-\alpha^{2}\right)^{1 / 2}}{4 \pi\left(\sigma_{h} \sigma_{l} A\right)^{1 / 2}} .
$$

Comparing eq (22) for an isotropic medium in which $\Omega=(2 \pi \sigma)^{-1}\left(1 / R_{0}-1 / d\right)$, with the equivalent isotropic expression obtained from eqs (22), (23), and (24), the apparent resistivity, $\rho_{a}$, is given by

$$
\rho_{a}=\frac{\sin ^{-1}\left(1-\alpha^{2}\right)^{1 / 2}-\sin ^{-1} \frac{R_{0}\left[2\left(1-\alpha^{2}\right)\right]^{1 / 2}}{K d}}{R_{0}\left[\sigma_{h} \sigma_{l}\left(1-\alpha^{2}\right)\right]^{1 / 2}\left[1 / R_{0}-1 / d\right]}
$$

The two extreme values of $K$ occur when the electrode axis is perpendicular and parallel to the $y$ axis. In these cases, we have

$$
\begin{gathered}
K_{\phi=0}=(2)^{1 / 2} \\
K_{\theta=\pi / 2, \phi=\pi / 2}=\left[2 \alpha^{2}+2\left(\frac{R_{0}}{d}\right)^{2}\left(1-\alpha^{2}\right)\right]^{1 / 2} .
\end{gathered}
$$

Insertion of eq (26) in eq (25) gives the extreme values of $\rho_{a}$, hence some estimate of the mean of $\rho_{a}$ over all angles. The latter quantity can be obtained more precisely by numerical integration of eq (25) over $\theta$ and $\phi$. When $R_{0} / d<<\alpha$, the terms in $K$ containing $R_{0} / d$ can be set to zero and the calculation of the mean considerably simplified. Finally, by taking the ratio of eq (22) at the extreme values, an expression containing $\alpha$ as the only unknown can be obtained and from this $\sigma_{l}$ and $\sigma_{h}$ determined.

These results were obtained as part of a general investigation of tissue resistivity carried out in collaboration with Dr. J. A. Abildskov. The work was supported by Research Grant H-3241 from the National Heart Institute of the National Institutes of Health, Public Health Service, and by a grant from the Heart Association of Onondaga County. The author also wishes to express his appreciation to Dr. Richard McFee for assistance in formulating the problems treated and for helpful manuscript criticism. 